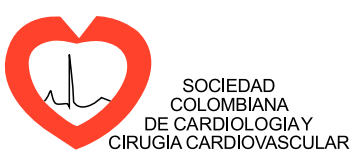

\title{
EDITORIAL
}

\section{The role of the new oral anticoagulants in the treatment of coronary disease}

\section{Papel de los nuevos anticoagulantes orales en el tratamiento de la enfermedad coronaria}

\author{
Hernando del Portillo ${ }^{\mathrm{a}}$, Juan J. Badimón ${ }^{\mathrm{b}}$, Jaime R. Cabrales ${ }^{\mathrm{a}}$, Darío Echeverri ${ }^{\mathrm{a}, *}$ \\ a Department of Hemodynamics and Cardiovascular Interventionism, Fundación Cardiolnfantil - Instituto de Cardiología - \\ Department of Medicine, Universidad del Rosario, Bogotá, Colombia \\ ${ }^{\mathrm{b}}$ Atherothrombosis Research Unit, The Zena and Michael A. Wiener Cardiovascular Institute, Icahn School of Medicine at Mount \\ Sinai, New York, United States
}

Received 30 September 2016; accepted 6 October 2016

Available online 17 November 2016

The new oral anticoagulants are here to stay in the prevention of ischemic cerebrovascular accidents in patients with nonvalvular atrial fibrillation. Several clinical studies have established their efficacy and safety ${ }^{1-3}$. However, the role these new oral anticoagulants might have in other pathologies, such as coronary disease, has not been well studied and there are still several important questions which have not been answered. One of these questions is the role the new oral anticoagulants could have in the secondary prevention of coronary disease, given that in spite of optimal medical treatment and dual anti-platelet therapy, the risk of reinfarction has only decreased $30 \%$. This phenomenon may possibly be explained by other related factors, such as lipid metabolism, the inflammatory state, and the prothrombotic state, in which the activated $X$ factor $(X a)$, plays a fundamental role by triggering the conversion of inactive prothrombin into thrombin, which is the strongest agonist for platelet aggregation ${ }^{4,5}$. The second question is related to those patients with nonvalvular atrial fibrillation and coronary disease who require stent implantation, in whom the

DOI of original article:

http://dx.doi.org/10.1016/j.rccar.2016.10.032

* Corresponding author.

E-mail address: decheverri@cardioinfantil.org (D. Echeverri). new oral anticoagulants could be an alternative in combination with antiplatelet medications.

Currently in Colombia, there are two types of new oral anticoagulants available: direct inhibitors of activated factor $X$ (rivaroxaban and apixaban) and direct thrombin inhibitors (dabigatran), which may be new therapeutic tools to answer these questions. Factor Xa inhibitors indirectly suppress the synthesis of thrombin by inhibiting this factor, while the direct antithrombin agents inhibit the activity of thrombin.

\section{First scenario: secondary prevention in coronary disease}

Coronary thrombosis depends on platelet activity and its integration with the coagulation pathways, where the vitamin K-dependent factors (II,VII, IX, and X) play a very important role ${ }^{6}$; this is why vitamin $\mathrm{K}$ antagonists (VKA) were studied a few years ago for secondary prevention of cardiovascular events, significantly reducing episodes of cerebrovascular accidents and myocardial infarction. The enormous benefits of this class of agents had an enormous disadvantage, which was the significant increase in bleeding ${ }^{7}$. This increase is probably associated with the difficulty in achieving and maintaining INR levels within 
therapeutic range, due to multiple interactions with other medications and even with foods, as well as a slow onset of action. All these conditioning factors are part of the reason why VKA are not currently considered first choice medications for the prevention and treatment of cardiovascular events.

Recent studies have evaluated the therapeutic potential of the new oral anticoagulants for secondary prevention in patients with coronary disease. The most important studies are APPRAISE-2 with apixaban ${ }^{8}$, and the ATLAS-ACS 2 TIMI 51 with rivaroxaban ${ }^{9}$, whose primary objective was to decrease cardiovascular death, myocardial infarction, and CVA in patients with known coronary disease. Unfortunately, the results were not very encouraging. APPRAISE- 2 was prematurely terminated due to excessive bleeding. The ATLAS-ACS 2 TIMI 51 study was positive, but the results are hard to explain from a pharmacological point of view, since the low doses of rivaroxaban at $2.5 \mathrm{mg}$ every 12 hours decreased cardiovascular mortality, but not myocardial infarction (which is the principal cause of cardiovascular death); while the $5 \mathrm{mg}$ dose every 12 hours reduced the rate of myocardial infarction but not cardiovascular mortality. In addition, both doses showed increased bleeding compared with the reference control group.

However, the idea that stands out to us, and which we consider important to highlight, is in the field of secondary prevention, since the benefit was mainly conferred by the results attained after the first year of treatment, the point at which most patients discontinue dual antiplatelet therapy. This is why we ask ourselves, "Will rivaroxaban be the next medication of choice for secondary prevention in coronary disease? In an effort to answer this question, the GEMINI-ACS-1 phase II study ${ }^{10}$ is currently underway, which will use rivaroxaban doses of $2.5 \mathrm{mg}$ every 12 hours in place of acetylsalicylic acid combined with clopidogrel and ticagrelor, seeking a double mechanism of action, and whose principle objective will be to evaluate safety in terms of bleeding, measured using the TIMI scale.

\section{Second scenario: Triple therapy with new oral anticoagulants}

With the changes in the population pyramid which entail an increased patient age, as well as the increased incidence of atrial fibrillation and clear benefit of anticoagulation with new oral anticoagulants in patients with atrial fibrillation, it is increasingly more common for patients to require dual antiplatelet therapy and anticoagulation. With regard to triple therapy in patients with atrial fibrillation and coronary disease plus percutaneous coronary intervention $(\mathrm{PCI})$, there are currently several ongoing studies using different types and doses of new oral anticoagulants, such as REDUAL PCI with dabigatran, PIONEER-atrial fibrillation with rivaroxaban, and AUGUSTUS with apixaban ${ }^{11}$, which will evaluate the combination of these new oral anticoagulants with the various $P_{2} Y_{12}$ receptor inhibitors. These studies are quite logical if three aspects are considered: 1 ) the greatest benefit of new oral anticoagulants versus VKA is a lower risk of bleeding; 2) some new oral anticoagulants have already been studied for secondary prevention; and 3) the new oral anticoagulants already have antidotes, some in phase III of research.

\section{New and possible scenarios of new oral anticoagulants in coronary disease}

In cases of ischemic heart failure and a reduced ejection fraction, a phase three study, COMMANDER-HF ${ }^{12}$, is in progress, which is based on the theory of thrombin as the critical element for worsening heart failure. Consequently, rivaroxaban $2.5 \mathrm{mg}$ every 12 hours plus dual or single antiplatelet therapy will evaluate the outcome consisting of mortality due to any cause, myocardial infarction and cerebrovascular accident. In this same context, but in the presence of left ventricular thrombi, cases have already been reported using new oral anticoagulants with complete resolution of the thrombus during echocardiographic followup $^{13}$.

Finally, in coronary ectasia, slow coronary flow, endothelial lesions and a pro-inflammatory state have been shown, which create a hypercoagulability environment ${ }^{14}$. Thus, as they fulfill the still accepted characteristics of Virchows triad, these patients would have a high predisposition for thrombus formation and the recurrence of myocardial ischemic events. In this coronary ectasia with slow flow and coronary thrombosis, different treatment strategies have been used, such as manual thrombectomy plus anticoagulation, intracoronary thrombolytics, acetylsalicylic acid plus warfarin, glycoprotein $\mathrm{II}_{\mathrm{b}} / \mathrm{III}_{\mathrm{a}}$ inhibitors, and drug eluting stents, yielding controversial results ${ }^{15}$. In light of this, a dose of rivaroxaban of $2.5 \mathrm{mg}$ every 12 hours could be an interesting and viable pharmacological option for this group of patients, since there is as yet no known specific treatment for the management of this pathology. The use of new oral anticoagulants in this context could be a new field to explore in clinical research.

\section{References}

1. Patel MR, Mahaffey KW, Garg J, Pan G, Singer DE, Hacke W, et al. Rivaroxaban versus warfarin in nonvalvular atrial fibrillation. $\mathrm{N}$ Engl J Med. 2011;365:883-91.

2. Granger CB, Alexander JH, McMurray JJV, Lopes RD, Hylek EM, Hanna $M$, et al. Apixaban versus warfarin in patients with atrial fibrillation. N Engl J Med. 2011;365:981-92.

3. Connolly SJ, Ezekowitz MD, Yusuf S, Eikelboom J, Oldgren J, Parekh A, et al. Dabigatran versus warfarin in patients with atrial fibrillation. N Engl J Med. 2009;361:1139-51.

4. Vilahur G, Badimon JJ, Bugiardini R, Badimon L. Perspectives: The burden of cardiovascular risk factors and coronary heart disease in Europe and worldwide. Eur Heart J. 2014;16 suppl A:A7.

5. Santos-Gallego CG, Badimon L, Badimón JJ. Perspectives: Direct and specific inhibition of factor $\mathrm{Xa}$ : an emerging therapeutic strategy for atherothrombotic disease. Eur Heart J. 2014;16 suppl A:A56.

6. De Caterina R, Husted S, Wallentin L, Andreotti F, Arnesen H, Bachmann F, et al. Oral anticoagulants in coronary heart disease (Section IV). Position paper of the ESC Working Group on Thrombosis-Task Force on Anticoagulants in Heart Disease. Thromb Haemost. 2016;115:685-711. 
7. Hurlen M, Abdelnoor M, Smith P, Erikssen J, Arnesen H. Warfarin, aspirin, or both after myocardial infarction. N Engl J Med. 2002;347:969-74.

8. Alexander JH, Lopes RD, James S, Kilaru R, He Y, Mohan P, et al. Apixaban with antiplatelet therapy after acute coronary syndrome. N Engl J Med. 2011;365:699-708.

9. Mega JL, Braunwald E, Wiviott SD, Bassand J-P, Bhatt DL, Bode $C$, et al. Rivaroxaban in patients with a recent acute coronary syndrome. N Engl J Med. 2012;366:9-19.

10. Povsic TJ, Roe MT, Ohman EM, Steg PG, James S, Plotnikov A, et al. A randomized trial to compare the safety of rivaroxaban vs aspirin in addition to either clopidogrel or ticagrelor in acute coronary syndrome: The design of the GEMINI-ACS-1 phase II study. Am Heart J. 2016;174:120-8.

11. Heidbuchel $H$, Verhamme $P$, Alings $M$, Antz $M$, Diener $H-C$, Hacke W, et al. Updated European Heart Rhythm Association Practical Guide on the use of non-vitamin K antagonist anticoagulants in patients with non-valvular atrial fibrillation. Europace. 2015;17:1467-507.
12. Zannad F, Greenberg B, Cleland JGF, Gheorghiade M, van Veldhuisen DJ, Mehra MR, et al. Rationale and design of a randomized, double-blind, event-driven, multicentre study comparing the efficacy and safety of oral rivaroxaban with placebo for reducing the risk of death, myocardial infarction or stroke in subjects with heart failure and significant coronary artery disease following an exacerbation of heart failure: the COMMANDER HF trial. Eur J Heart Fail. 2015;17:735-42.

13. Mano Y, Koide K, Sukegawa H, Kodaira M, Ohki T. Successful resolution of a left ventricular thrombus with apixaban treatment following acute myocardial infarction. Heart Vessels. 2016;31:118-23.

14. Brunetti ND, Salvemini G, Cuculo A, Ruggiero A, De Gennaro L, Gaglione A, et al. Coronary artery ectasia is related to coronary slow flow and inflammatory activation. Atherosclerosis. 2014;233:636-40.

15. Eitan A, Roguin A. Coronary artery ectasia: new insights into pathophysiology, diagnosis, and treatment. Coron Artery Dis. 2016;27:420-8 